\title{
INTERACTION COMMERCE, A TECHNOLOGICAL ARCHITECTURE FOCUSED ON RECOMMENDER SYSTEM
}

\author{
Luca Salvatori \\ University of Camerino \\ Via del Bastione 1, 62032, Camerino, Italy \\ luca.salvatori@unicam.it \\ Fausto Marcantoni \\ University of Camerino \\ Via del Bastione 1, 62032, Camerino, Italy \\ fausto.marcantoni@unicam.it
}

\begin{abstract}
This paper aims at introducing a type of social commerce architecture to which the name Interaction Commerce has been given. First, a global description of the main macro-components forming the structure of this architecture is provided. Such components also take care of managing e-commerce activities and social relationships within the architecture. Second, the focus is set on the analysis of the single components that are key to the social aspects of the architecture. A special chapter is then entirely focussed on a topic that is considered extremely important by the entire research community, i.e., recommender systems. After providing a general introduction on the topic, the two most common recommendation approaches are analyzed and compared. These are the content-based approach and the collaborative filtering approach. The analysis has shown how all recommender systems are threatened by the cold-start problem. Studying recommender systems has allowed for their implementation in the architecture, which now has a new "social" approach that is able to solve the new user cold-start problem. An architecture prototype was developed and tested in order to be validated.
\end{abstract}

Keywords: Social Commerce Architecture, Electronic Commerce Architecture, Recommender System Architecture, Recommender System with Social Network 


\section{INTRODUCTION}

With the advent of social media, particularly of social networks, people spend increasingly more time in front of some kind of device in order to communicate and exchange information, ideas, or data pertaining to their work and also personal environment ${ }^{1,2}$. We are truly witnessing a quick and radical change in how the Internet is used. This change also affects e-commerce platforms that are almost forced to adapt and meet their customers' new needs. This innovative current has benefitted the consumer but also the seller who now has the opportunity to increase its volume of sales $^{3}$.

Currently companies prefer not to use the old general and massive advertising techniques but prefer to establish a relationship with their customers in order to offer recommendations specifically aimed at their buying habits. Before deciding on the purchase of a product, customers try to establish contact with other users paying attention: for example, to comments left, preferably someone known to them. In short, the customers are becoming participating content writers in order to communicate and exchange opinions ${ }^{4}$. According to many other researchers, social activities may lead to increased sales due to the creation of stable relations of trust among the actors ${ }^{3,5}$.

In the last few years, the above-mentioned phenomenon has been expanding considerably, becoming a significant opportunity for theoretical and practical research. This phenomenon is called social commerce and refers to e-commerce activities that lead to online purchases, using social media and Web 2.06 . This system enhances the potential of e-commerce, increasing the user's opportunity for social relations through the use of social media. Some experts in this sector and researchers consider e-commerce as collaboration amongst customers aimed at finalizing purchases in an environment similar to a social network ${ }^{2,7}$. Others offer an interpretation stemming from the sellers' viewpoint and define it as collaboration of sellers to achieve business advantage ${ }^{8,9}$. Even though the literature offers many definitions of this phenomenon, all concur that the most important difference between e-commerce and social commerce is that, in e-commerce, users decide alone on their purchases, while in social commerce they interact with the community thanks to the tools offered by the platform.

In parallel with studies on e-commerce and later on social commerce, particular attention was given by the researchers to the topic of recommender systems. These systems have evolved as much as the phenomenon of social commerce because their purposes is to offer 
personalized support to filter and order the large amount of information available, according to the customers' interests and tastes. The recommender system can be defined as a system that guides the user through a large number of options to allow him or her to find elements to suit its needs and interests ${ }^{10}$. It provides a platform that can guarantee finding the right knowledge, within the right context, for the right person, and in the right amount $^{11}$.

The scope of this paper, as well as the reason for its writing, is to offer a description of social commerce architecture. According to the results of current studies, there is a lack of articles that provide an innovative structure that could be implemented for social commerce, one that could help researchers and those who need to implement such a platform. To go into detail, this work would like to demonstrate how this approach can, among other advantages, help to better optimize how all the components work together. On this subject, it describes the advantages that a recommender system can obtain when inserted into a social environment. If, in fact, on one hand, it is possible to build a valid recommender system by knowing the ratings (implicit or explicit) that a user gives to a product within the social commerce platform; on the other hand, the recommender systems can be utilized from the first time the user accesses the platform, by acquiring from the social network its tastes and aptitudes. It is then possible to limit the cold-starting problem that plagues all e-commerce and social commerce. Ultimately, the aim of this work is to provide an innovative and proved way to resolve the new user cold-start problem.

The article is structured as follows. Section 2 shows the architecture of social commerce, providing an overview of its components and offering a detailed description of each. Section 3 analytically describes the recommender system components, starting with its general concepts, then to reach implementation choices and show the innovative character of this research. Section 4 analyzes the workflow of a hypothetical user within the described architecture. Section 5 shows the results of the tests done to verify the recommender system's components functionality. Section 6 provides the findings of the work.

\section{INTERACTION COMMERCE ARCHITECTURE}

\subsection{Description of the Architecture}

The interaction commerce structure (Figure 1) is modular and shows two macro-components: the e-commerce management component and the social relationship management component. In detail, the two 
macro-components show the various micro-components. The e-commerce management component includes the catalogue, cart, discount, customer, seller, checkout, order, shipping, and payment components. The social management component shows the management of the wish-list, recommendation system, transaction ratings, product reviews, and communication.

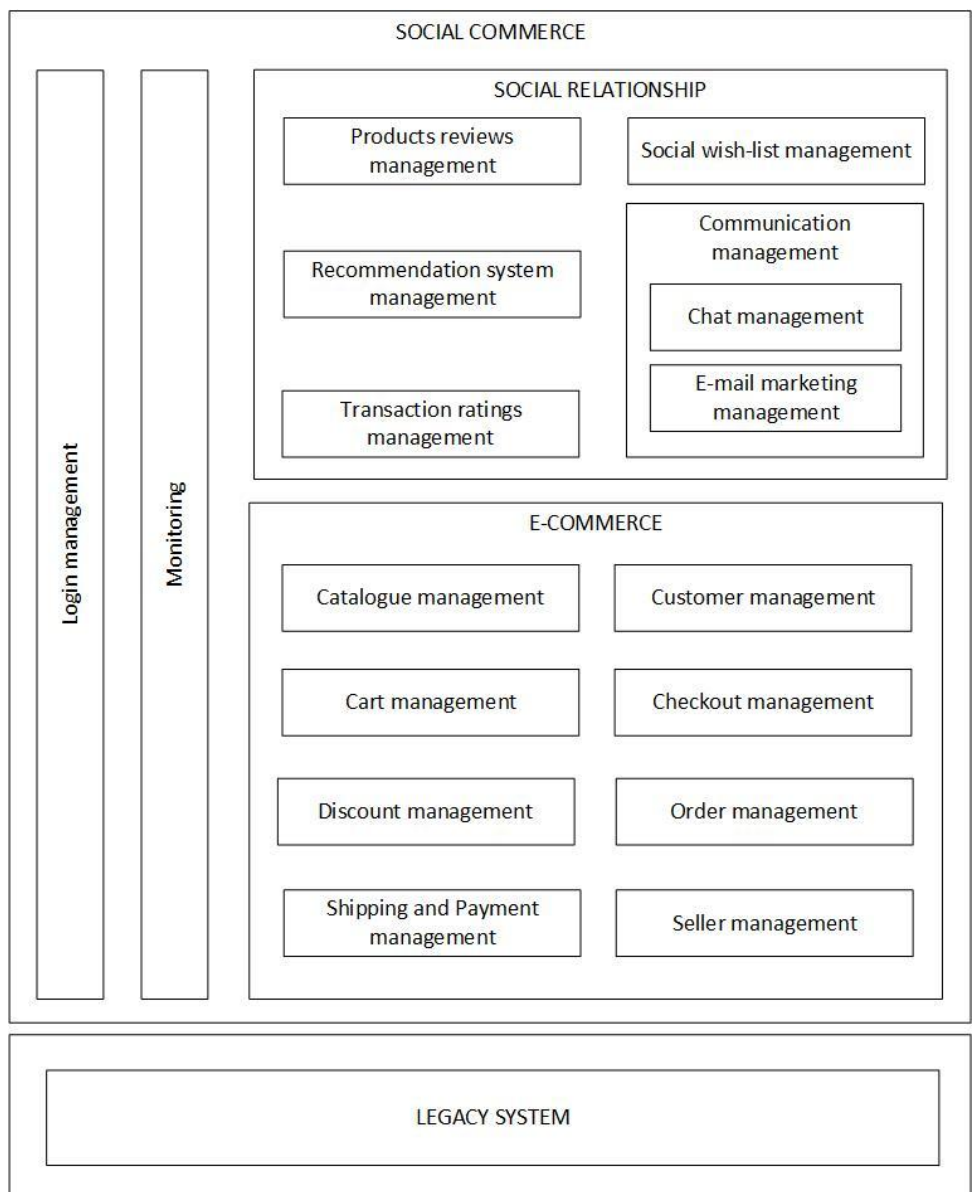

Figure 1. Interaction commerce architecture

Next to these two macro-components there are two independent components, the login and monitoring management components, which relate to the e-commerce as well as the social components. On a lower level, we find the legacy management component that includes all current business information relative to products, warehouses, and customers. It is important to remember that the e-commerce management component represents the typical and fundamental component of any e-commerce system. The evolution of such a system is represented by an innovative component: the 
social relationship management component that manages the social interaction between the various users (sellers and buyers). These relationships are possible because of micro-components that allow users to interface with each other creating a sort of community. Within such a community, users exchange information, thus creating a social presence based on mutual trust. The result is the user's higher propensity to make a purchase. The buyers have more trust in the store and consider the product's characteristics reliable. Within the various micro-components provided by the social relationship management component is the recommender system management component. It allows the buyer to receive suggestions focused on potential products through the use of dedicated algorithms. The selection of the suggested items is the result of the study of the relationship between buyers and between buyers and products.

\subsection{Analysis of the Individual Components}

Within a social commerce platform, the figures of customer and seller play absolutely primary roles. These actors interact with the platform in a consistent way, taking full advantage of its potential. The customer, in particular, benefits from the social and buying roles the platform offers, while the benefits enjoyed by the seller, as a user, come from the potential sales deriving from the platform. This last stakeholder is interested in selling as a way to increase profits as well as increase visibility. In order to do so, the seller needs to know the customers very well, particularly their tastes. By collecting such information, the seller will know which of its products are of more interest.

The e-commerce macro-component includes the catalogue, customer, seller, cart, checkout, order, discount, and shipping and payment management components. Some of these components are useful only to the customer, others only to the seller, and others are useful to both. The analysis of the e-commerce micro-components is not provided because they only consist of e-commerce basic and usual components about the system itself. Within the social relationship macro-component, we can find the following micro-components: the social wish lists, recommendation system, product reviews, transaction ratings, and the communication management components.

Within the social context, the social wish list management component manages activities relevant to the wish list from the sellers and the customers. The wish list is a list of specific products and allows the customer to keep track of the products it desires. Particularly, by managing the wish list, the customer can create new lists, delete an existing one, and add or delete products wished for, as indicated in the list. The wish list is not 
only useful to a customer for personal and private purposes, it also allows the customer to share it with other customers, so they can be aware of the products the originating customer is interested in and desires. Similarly, the seller, by viewing the wish list, can monitor customer's preferences and anticipate market trends. This component is connected with the catalogue management and recommender system management components. By interfacing with the catalogue management component, it can retrieve the details of the products that are part of the social wish list. The connection with the recommender system management component means that the presence, on social wish lists, of certain products desired by the customers may influence the products' recommendation.

The recommender system management component, which represents the most innovative part of this paper, and will be further studied in depth in a separate section, consists of two main activities: data collection and data extraction, each carried out by different components. Data collection gathers and collects data about the relationship among customers or between customers and products. Data extraction selects the elements meeting a customer's tastes and preferences. The recommender system management component uses a collaborative approach through the use of collaborative filtering type algorithms. To solve, at least partially, the cold-start problem, this component uses the social information of the logged-in user by means of one of the enabled social networks. By doing so, it is possible to know the tastes and interests of a newly registered user. This component interfaces with the login, wish list, product reviews, communications, and orders management components. These connections originate from the component's need to know the client's tastes, preferences and tendencies, in order to suggest appropriate products of interest. Another important connection is with the e-mail marketing management component, which receives the products to suggest and advertise to the client.

The communication management component organizes sharing of client and seller information stimulating the customer and making it easier. This component has two characteristic activities: chat management and e-mail marketing. Thanks to chat management, an online purchase becomes similar to an offline purchase done in a real shop. This helps the customer find the desired products by communicating with other customers about the products' characteristics, asking for suggestions, sharing opinions, and comparing their purchasing experience. Because it comprises all customer and seller e-mail activities, e-mail marketing management allows customers to sign up for a mailing list, and sellers to manually add a new customer. In this way, sellers are always in touch with customers and can inform them of discounts, special sales, or the arrival of a new product. In regards to the 
connections with other components, the chat management component ties in with the customer and seller management components because it is necessary to let the user know that there are other customers or sellers available to use the chat. The newsletter management component interfaces with the catalogue, discounts, and recommendation management components. The connection with the catalogue management component is necessary to allow sellers to add automatically, in the e-mail, details about a product and the pertinent web page. The discount management component communicates to the e-mail marketing management component the discounted products, while the connection with the recommender management component determines which products to suggest in a marketing e-mail.

The products review management component allows a client to create user-generated content: the consumer processes a particular product's reviews on a five-star scale. The reviews left by other customers, and visible to other users, play an important role in influencing a customer during the choosing of a product. This component connects with catalogue and customer management components because it needs to know the identity of the user generating a certain review as well as knowing to which products it is associated. A further connection is with the recommender system management component, which receives information about the user's preferences and is then able to suggest targeted products.

The transaction rating management component attends to managing reviews and the scores generated by customers about a certain purchase transaction. The topics generally found in a review relate to the seller's reliability, shipping speed, payment security, and product quality. Customers can express a personal opinion on these aspects of a transaction, influencing and stimulating other customers to buy via the social commerce platform. Sellers can also view customer-generated reviews, decide to accept them and make them public, or reject them. By considering the scores attributed to transactions, sellers can know the customers' level of satisfaction toward the platform and the problems it might present. This way sellers can fill any gaps and resolve problems, responding to the customers' needs in an effective manner. The transaction rating management component interfaces with the order management component to connect a customer's personal opinion or score to a specific order.

Next to the two macro-components, e-commerce and social relationship, we can find two other independent elements: the login and the monitoring management components. 
The login management component authenticates the users. In fact, they can be recognized by the system by inserting their username and password. Alternatively, they can login directly through the main social networks, which can offer some advantages to the customer. Through Facebook and Twitter, the user can $\log$ into the system without inputting further registration data and validation of its e-mail address. Using external authentication providers, the user can transparently register anew and have a login, speeding the procedure, while at the same time increasing the security. This is possible because, during the authentication process, no password is transmitted from the authentication provider to the social commerce. In case of successful authentication, the users can access the platform as customers or sellers, to carry out all the operations enabled by their profile. Should the authentication fail, the user will receive a warning message from the system containing a description of the error as well as the opportunity to input its credentials again. This component interfaces with the consumer and seller management components, receiving from them the login data. In order to perform certain operations within the system, the customer needs to be authenticated. It becomes necessary, for example, to view the list of confirmed orders, to use the chat, to enter a rating about a transaction or a rating or comment about any product, and to manage the social wish list. It is easy to see how the login management component is connected to the components managing these activities. Authentication is a fundamental activity for sellers in order to perform administrative tasks such as managing other sellers, customers, catalogues, discounts, the newsletter, payment and shipping modes, orders, chats, transaction ratings, recommendation system, and social wish-list and to perform monitoring activities. It is important to note how this component is connected to the recommender system management component. As illustrated in Chapter 3, via "social login," it is possible, having the customer's prior authorization, to export information pertaining to that customer in order to improve the customization of products that might appeal to the customer's tastes and, particularly, to limit any cold-start problems that plague the vast majority of recommender systems.

The monitoring management component can well be considered as the component that is most significant for the seller. By monitoring customers' preferences and needs, the seller is more aware of the type of customers interacting on the platform and their needs. So the seller can establish a relationship of trust with its customers and anticipate market trends. By providing transparency and safety, this component plays an essential role in guaranteeing the platform's reliability to the users. Quite frequently social commerce and social networking sites are, in fact, subjects of social engineering attacks that violate the terms of safety and privacy. This is 
mostly due to the intense sensible data exchange activity that happens on public and private workspaces. The monitoring management component interfaces with almost all other architectural components because its role is to supervise social commerce activities.

\section{RECOMMENDER SYSTEM}

The abundance of information available online, often superfluous, paired with its dynamic and heterogeneous nature, increases the difficulty of easily finding what is wanted, at the needed time, and according to certain requirements. This creates the need to access personalized information: the user needs personalized support to filter and order the large amount of information available, according to the user's interests and tastes. The purpose of the recommender system is to provide personalized products and services and to recommend them to the user. This way the problem of ever-increasing online information can be managed, thus improving seller-customer relationships ${ }^{12}$. Finally, within the social commerce environment, the recommender system must be capable of suggesting to the user a product meeting its interests.

Historically, prior to 1990 , researches about the recommender system were aimed at retrieving information and managing search filters ${ }^{13}$. Later, after finding out that evaluation systems were the direct result of the voting system, recommender systems emerged as a research field in its own right ${ }^{14}$. It is also important to note how recommender systems were developed in close contact and in parallel with the $\mathrm{Web}^{15}$. During the last 20 years, the topic of recommender systems has represented a very active research field ${ }^{16}$. This is the result of the many ways it is employed by various domains, such as business and education. Given the extent of the subject, the various objects and application methodologies, the recommender system can also deal with marketing, information technology, information science, computer science, and management ${ }^{17}$. Thanks to social and commercial factors directly influenced by the recommender system, it has become an important academic and industrial research area ${ }^{18}$.

Through a series of aggregations and analysis of advice coming from other users, the recommender system can recognize products of significance to the user. These opinions can come from accredited entities or common users, considering that the advice coming from the latter generally seem to be more appreciated. Thanks to the use of analysis systems, it is possible to know the probability that a product may be of interest to the user and that the user might wish to purchase it. In this instance, a personalized suggestion can be made to the user ${ }^{17}$ about the most appropriate product. 
The recommender systems can be viewed by businesses as useful tools, especially for those operating in e-commerce, to obtain a competitive advantage. A system that produces positive recommendations can inspire the customer to trust the company to find the products for which it is truly searching. The recommender system also can allow the company to know the customers' preferences and plan ad hoc offers individually tailored to each customer ${ }^{19}$. For some time we have been seeing the development of social media and more generally of the Web 2.0, which allow users to actively participate and create and share new content. This is the linchpin of the research done to demonstrate how using social networks to support recommender systems bring many benefits to the recommended generating algorithm, notably limiting the cold-start problem.

Recommender systems can be generally classified as content-based filtering $(\mathrm{CB})$ or collaborative filtering $(\mathrm{CF})$. The latter does not need to compete with content-based filtering systems; in many instances, both recommendation technologies are integrated to provide a powerful hybridized solution.

\subsection{Content-Based Approach}

Content-based recommender systems recommend to the user products based on what the user preferred in the past, considering the type and characteristics of the product at that time ${ }^{19}$. The recommender systems must know which products are in the catalogue, the users, and the relationship between a user and a product. Content-based recommendation techniques suggest items or merchandise similar to products previously preferred by a specific user ${ }^{20}$. The algorithm is supported by a vector containing the weights associated with particular keywords. The products likely to be recommended are compared with those that previously received a positive rating by the user. By studying products' similarities, it is possible to deduce and then suggest the most useful ${ }^{21}$.

The systems implementing a content-based recommendation approach analyze documents, and/or the description of elements previously rated by the user, and build a model or profile of the user's interests based on the characteristics of the products that the customer rated ${ }^{22,23}$. A user's profile automatically outlined by the recommender system can be quite varied and dependent on the learning methods applied. It consists of a structure representing the user's interests, and it is used to recommend new products useful to the user. The process of recommendation compares the user's profile attributes with those of the product ${ }^{24}$. The result is an estimation of the user's interest level in the specific object. 
The recommendation process consists of three distinct steps ${ }^{24}$. The first step is to prepare useful information that will be used in successive steps. Some of the information might not have a clearly delineated structure (for example, the product description in social commerce) and require some preprocessing to extract the structure of significant information and to present it in an appropriate form that is suited to the work carried out by later activities. The second step generates the user profile analyzing and generalizing data structured by the content analyzer, often using machine learning techniques. This information includes a specific user's preferred products and is used to determine the most significant common features (preferences) that can be used to distinguish such products ${ }^{20}$. The last action is the comparison between the potentially recommendable product's features and the user's profile, using similarity parameters. The product will be recommended if it shares the user's features ${ }^{20}$. It is possible to deduce that a user's profile capable of reflecting the user's preferences increases the efficacy of the information access process.

\subsection{Collaborative Filtering Approach}

One of the most common techniques for generating recommendations through a recommender system is the collaborative filtering approach. ${ }^{11}$ There can be different approaches, however. One of these approaches is user-based type; another is item-based type, but, because the latter is used only for performance reasons or if there are millions of users, we will only refer to the user-based collaborative filtering approach. This technique is used by recommender systems to anticipate the usefulness of an element to the user according to the similarities it shares with other customers. Recommendation techniques based on collaborative filtering, in order to recommend products appealing to a specific user, use data (generally opinions) gathered from other people having similar interests ${ }^{25}$. The basis for a collaborative filtering recommendation system ${ }^{26,27}$ is the users' capability to provide ratings about a group of elements. In this manner, a system holding sufficient data can generate recommendations for any user based on information received from similar customers. Every user in the collaborative recommender system rates only the products it knows, thus creating an interest's profile. Subsequently, the recommender system pairs the subject user with others having similar interest and tastes. In this way, ratings from similar users are used to generate recommendations for the user $^{28}$. It is inferred that collaborative-type recommender systems anticipate the user's interest in a product based on decisions reached by analyzing the individual and not using a machine analysis of content ${ }^{29}$.

Collaborative filtering techniques use a database made up of preferences generated by users about products that could appeal to another 
user. The intent of these preferences is to predict further products. In a typical collaborative filtering scenario there is a list of $m$ users $\left\{u_{1}, u_{2}, \ldots, u_{\mathrm{m}}\right\}$, and a list of $n$ products $\left\{i_{1}, i_{2}, \ldots, i_{n}\right\}$. Each $u_{\mathrm{i}}$ user has a list of $I_{u i}$ products to which it associates, directly or indirectly, a rating (for example, a rating obtained by studying the user's behavior) ${ }^{30}$. It is important to note that the collaborative filtering approach is certainly to be considered an interesting open field of research ${ }^{31,32}$.

\subsection{Comparison of Approach Typologies}

Table 1 summarizes all the comparison factors among the content-based approach and collaborative filtering approach.

Table 1. Recommendation methods comparison

\begin{tabular}{|c|c|}
\hline Content-based approach & $\begin{array}{l}\text { Collaborative filtering } \\
\text { approach }\end{array}$ \\
\hline User independence & Dependence on community \\
\hline $\begin{array}{l}\text { Not affected by gray sheep } \\
\text { problem }\end{array}$ & $\begin{array}{l}\text { Affected by gray sheep } \\
\text { problem }\end{array}$ \\
\hline $\begin{array}{l}\text { Homogeneity values are } \\
\text { obtainable even if the data are } \\
\text { scattered }\end{array}$ & $\begin{array}{l}\text { Homogeneity values are } \\
\text { unobtainable if the data are } \\
\text { scattered }\end{array}$ \\
\hline $\begin{array}{l}\text { It is possible to justify the } \\
\text { choice of recommended } \\
\text { products }\end{array}$ & $\begin{array}{c}\text { It can be viewed as a black } \\
\text { box }\end{array}$ \\
\hline $\begin{array}{l}\text { Not affected by new } \\
\text { product cold-start problem }\end{array}$ & $\begin{array}{l}\text { Affected by new product } \\
\text { cold-start problem }\end{array}$ \\
\hline Better performance & Worse performance \\
\hline $\begin{array}{l}\text { Not considered easy to use } \\
\text { and successful }\end{array}$ & $\begin{array}{l}\text { Considered easy to use and } \\
\text { successful }\end{array}$ \\
\hline $\begin{array}{l}\text { Applicable only in certain } \\
\text { application contexts }\end{array}$ & $\begin{array}{l}\text { Applicable in all application } \\
\text { contexts }\end{array}$ \\
\hline $\begin{array}{l}\text { Risk of errors from } \\
\text { machine analysis }\end{array}$ & $\begin{array}{l}\text { No risk of errors from } \\
\text { machine analysis }\end{array}$ \\
\hline $\begin{array}{l}\text { Not able to distinguish } \\
\text { comparable or identical }\end{array}$ & $\begin{array}{l}\text { Able to distinguish } \\
\text { comparable or identical products }\end{array}$ \\
\hline products with different names & with different names \\
\hline $\begin{array}{l}\text { Not capable of managing } \\
\text { unexpected factors or events }\end{array}$ & $\begin{array}{c}\text { Capable of managing } \\
\text { unexpected factors or events }\end{array}$ \\
\hline
\end{tabular}


Adopting the content-based recommendation paradigm has many advantages if compared with the collaborative type. First, in order to find out which products a user is interested in, this paradigm utilizes only the ratings generated by the user in order to build its profile (user independence). Collaborative methods are influenced by the need to analyze the ratings from other users in order to find those who share interests and preferences with the subject user $^{13}$. Consequently, for example, the content-based approach is not affected by the so-called "gray sheep" problem, which is the problem arising when a user disassociates itself from a group and does not present preferences in common with others in the group $^{33}$. Similarly, in a collaborative filtering approach, homogeneity values are based on common products and are unobtainable if the data are scattered and if there are few products rated commonly by the users. Furthermore, by using a content-based system, the same problems should not appear when people give more positive results to their products and negative results to competing products ${ }^{30}$, negatively affecting other users' recommendations. A content-based approach clearly justifies the choice of recommended products by listing the characteristics of the recommended product. It is not possible to give the same reason when using a collaborative filtering approach. The latter can only justify the choice by stating that an element was recommended because an unknown user with similar taste used that product $^{24}$. A collaborative recommendation approach can only be viewed as a black box because, given its nature, it is not possible to fully know the reasons that spur the recommendation of one product over another. Thus the user cannot figure when to have confidence in a recommendation and when to not. Another point in favor of a content-based approach is the absence of the cold-start problem in a new product. This limitation affecting the collaborative-type approach lies in the ability to recommend a product to a user only if that element is associated with a substantial number of ratings. ${ }^{34}$ Generally a content-based approach performs better, especially when the number of users and/or products is high. In this instance, the collaborative filtering recommender system would manage a large and sparse user-product table, to the detriment of the performance and the system's computational resources ${ }^{25}$.

However, a collaborative approach should not be considered completely disadvantageous. In fact, it shows many positive elements when compared with the content-based one. First and foremost, the collaborative filtering approach is considered easy to use and very successful ${ }^{35,36}$. Above all, it does not need to know the application's domain in order to pair certain characteristics with a specific item to be recommended. The same cannot be said for content-based algorithms that, in order to provide satisfactory suggestions, require in-depth analysis of the products' information content. 
Only by clearly defining the item's characteristics will it be possible to determine which products the user likes ${ }^{37}$. The need for a content-based approach limits its utilization in certain fields and application contexts ${ }^{29}$. However, by using a collaborative approach, there is no need to incorporate the information about the product's characteristics ${ }^{28}$, and there is no risk of errors from machine analysis of content ${ }^{29}$. It must be said that, given its nature, this approach cannot distinguish comparable or identical products with different names. The latter problem could be circumvented by using the thesaurus to automatically expand the vocabulary, but this would create performance problems for the system ${ }^{38}$. In addition, the collaborative filtering recommender system can provide recommendations of more recent products more effectively than the other recommender approach, by virtue of its principle of recommendations based on the preferences of users presenting characteristics similar to the user in question. It is capable of managing unexpected factors or events. It would be difficult for a content-based recommender system to suggest products that would not meet the user's taste because the system analyzes the products' characteristics and similarities ${ }^{39}$. Lastly, by adopting a collaborative-filtering approach, it is possible to limit, albeit not in absolute, the problem of offering recommendations to new users. This problem makes it impossible to provide reliable recommendations to the new users for whom there are few data $^{40}$. Notwithstanding, it must be noted that collaborative filtering is not immune from such problems ${ }^{14,41}$.

After analyzing the pros and cons for each approach system, it was decided to use a collaborative filtering approach for this architecture. The choice was made mainly by considering the actual ability for that approach to work in all sectors of application. This appears to be effective regardless of the knowledge about a specific application domain. Therefore this is considered to be the most flexible of the two approaches, and easier to implement. Using that approach, which does not require finding similarities among the products sharing the same characteristics and attributes, there is no need to define the products' distinctive characteristics, a process that, in some fields of application, requires manual intervention in order to obtain satisfactory results.

\subsection{Recommender System's Architectural Analysis}

The recommender system component (shown in Figure 2) is structured as two subcomponents: the data collection management component, and the data extraction management component. The first relates to the acquisition of the user's implicit and explicit interests. The second relates to the processing of the user's preferences in order to find products of its liking, using data obtained from the other component. More specifically, the data 
collection management component is made up of five micro-components, each of which can receive the user's preferences from a different source. Each provides an explicit or implicit rating, with different degrees of positivity that the user attributes to a specific product.

In more detail, the purchased products collection management component acquires information about the products purchased by the user. The viewed products collection management component manages the user's preferences about a product according to the circumstance in which it was viewed or not viewed by the user. The function of the wish-list collection management component is to keep track of the products the user placed on its wish-list and thereby is interested in. The rated products collection management component acquires the user's explicit ratings. The liked products collection management component extracts the information about the products present in the social commerce catalogue and compares them with the products the user has expressed a preference for on the social network(s), in order to know if they are interesting or not to the user.

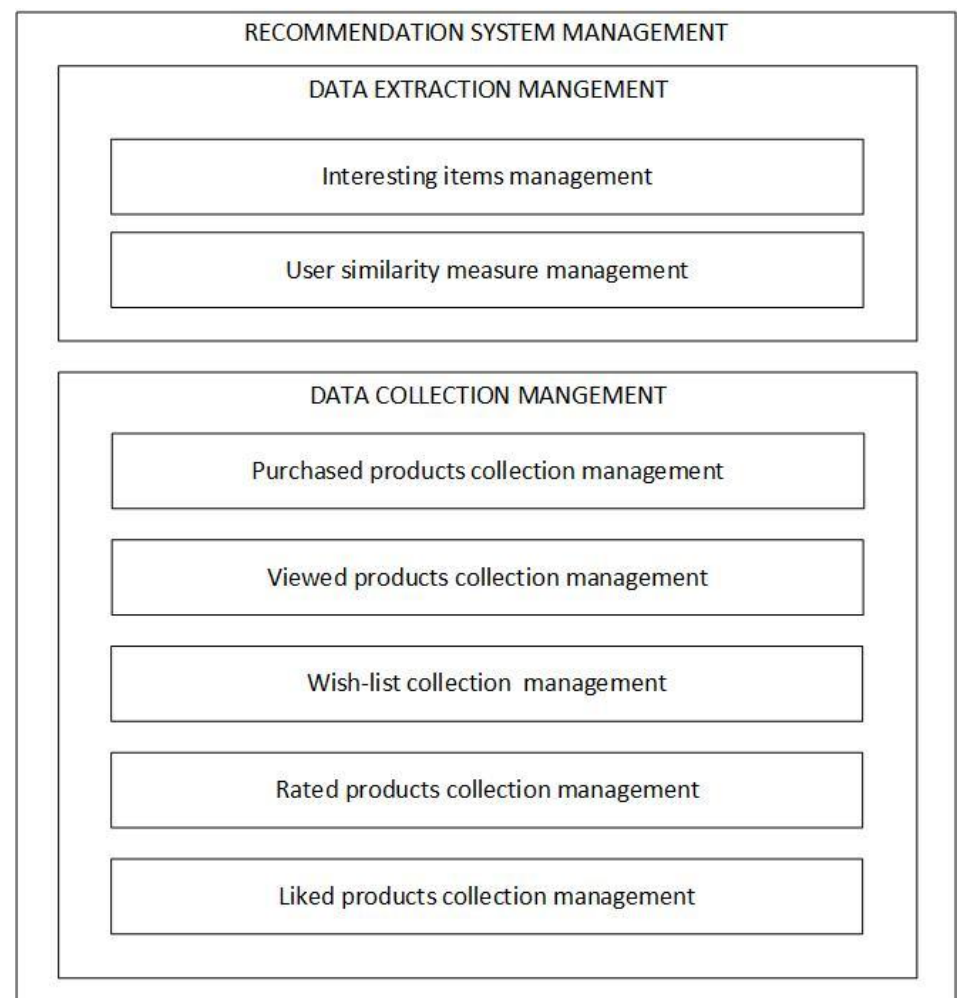

Figure 2. Recommender system's architecture 
By obtaining the user's preferences from various social networks, it is possible to limit and in some cases to resolve the user cold-start problem ${ }^{42}$, which is the inability to provide recommendations due to lack of initial ratings. The cold-start problem is of three different types: new community, new product, and new user ${ }^{34}$. Note that recommender systems that have been implemented in a platform for a long time are mainly troubled by the single user's cold-start problem ${ }^{43}$. The new community problem is found in the difficulty a recently started recommender system has to provide reliable information considering the lack of a sufficient number of ratings ${ }^{44}$. The new element problem is encountered when a product new to the recommender system has not yet been rated and is thus non-recommendable ${ }^{43}$. An item without recommendation is ignored by the users in the community who have no motivation to rate it. It is a vicious cycle in which some of the recommender system's elements have been taken out of the rating and the recommending process ${ }^{15}$. The new user problem ${ }^{45}$, ${ }^{46}$ has been for some time one of the most significant difficulties for an operating recommender system ${ }^{40}$. Within the social commerce platform, new users do not provide ratings that the recommender system can utilize, making it impossible for the system to offer the user personalized recommendations. We must consider the risk that users offering their initial ratings will decide to stop using the recommender system. Not receiving sufficient ratings to provide reliable recommendations, the system could suggest products of no interest to the user, and appear to be ineffective ${ }^{15}$.

A possible solution to the "new community" and "new element" cold-start type problems would be to have a group of users motivated to rate each new product. Instead, the usual solution to the new user cold-start problem is to encourage new users to choose ratings within the platform ${ }^{15}$. As an alternative to these approaches, a new solution has been developed. It recovers and utilizes information about the user gathered from external social networks in order to know the user's preferences and tastes about the products in catalogue, even in the absence of ratings. This way a user does not need to receive constant invitations to rate products in order to receive useful recommendations. A user's data are automatically received by the recommender system in a simple and transparent way.

The second component of the recommender system, the data extraction management component, is divided into two subcomponents. Each is occupied with different development steps in order to provide recommendations. Specifically, the user similarity measure management component finds similarities amongst users sharing preferences and tastes and provides them a list organized according their level of affinity, using a neighborhood-based approach. 
Given products $\boldsymbol{P}$ and group of users $\boldsymbol{U}$, each user's preferences $i \in U$ within all products $P$ are represented by an $R=S \times M$ matrix, where $S$ indicates the cardinality of the whole $U$, and $\boldsymbol{M}$ the cardinality of the whole $P$. This way, each element of the $r_{i, p} \in R$ matrix represents the user's preference as regards product $P$. The purpose of this component is to find the entirety of similar users. For each user $i \in U$, the complete set of similar users to whom will be offered recommendations is indicated by $N_{i} \subseteq U$.

According to the study of the literature and based on the amount and type of user data available, three distinct algorithms have been chosen for the user similarity measure management component: proximity-impact-popularity, Pearson correlation, and constrained Pearson correlation.

Proximity-Impact-Popularity. The proximity-impact-popularity (PIP) algorithm is used when there are few data on the user to generate recommendations because, in this case, it performs better than other similarity calculation processes ${ }^{47}$. In order to find similarities between two different users $\in U$ (namely, $I$ and $k$ ), this algorithm calculates four factors: Agreement, proximity, impact, and popularity. In particular, the product of the three last factors provides the similarity index.

\section{Agreement}

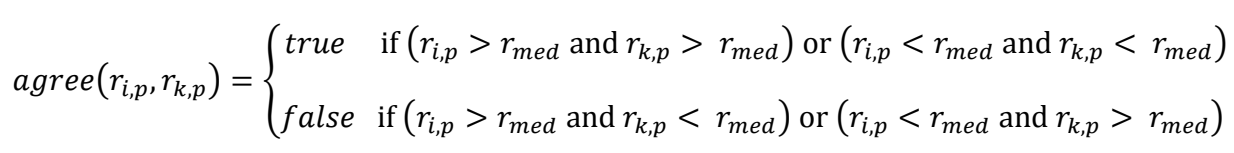

whereas

$$
r_{\text {med }}=\frac{r_{\text {max }}-r_{\text {min }}}{2}
$$

and where $r_{\max }$ and $r_{\min }$ represent, respectively, the maximum and minimum value of the scale in question.

\section{Proximity}

$$
\operatorname{prox}\left(r_{i, p}, r_{k, p}\right)=\left\{\begin{array}{cl}
\left|r_{i, p}-r_{k, p}\right| & \text { if } \operatorname{agree}\left(r_{i, p}, r_{k, p}\right)=\text { true } \\
2 \cdot\left|r_{i, p}-r_{k, p}\right| & \text { if } \operatorname{agree}\left(r_{i, p}, r_{k, p}\right)=\text { false }
\end{array}\right.
$$

\section{Impact}

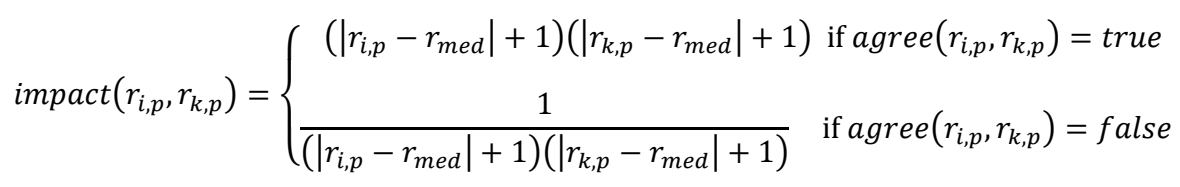




\section{Popularity}

$$
\operatorname{popul}\left(r_{i, p}, r_{k, p}\right)= \begin{cases}1+\left(\frac{r_{i, p}-r_{k, p}}{2}-u_{p}\right)^{2} & \text { if }\left(r_{i, p}>u_{p} \text { and } r_{k, p}>u_{p}\right) \text { or }\left(r_{i, p}<u_{p} \text { and } r_{k, p}<u_{p}\right) \\ 1 & \text { if }\left(r_{i, p}>u_{p} \text { and } r_{k, p}<u_{p}\right) \text { or }\left(r_{i, p}<u_{p} \text { and } r_{k, p}>u_{p}\right)\end{cases}
$$

where $u_{p}$ corresponds to all the users' average rating for a $\boldsymbol{p}$ product

\section{PIP Similarity}

$$
\operatorname{pip}_{i, k}=\operatorname{prox}\left(r_{i, p}, r_{k, p}\right) \cdot \operatorname{impact}\left(r_{i, p}, r_{k, p}\right) \cdot \operatorname{popul}\left(r_{i, p}, r_{k, p}\right)
$$

Pearson Correlation. The Pearson correlation similarity is used when there are sufficient data about the user to generate recommendations. By using their correlation, the rating similarity (rs) similarities between two different users $\in U$ (namely, $i$ and $k$ ) can be calculated, using the following formula:

$$
r s_{i, k}=\frac{\sum_{p \in P_{i, k}}\left(r_{i, p}-\bar{r}_{i}\right)\left(r_{k, p}-\bar{r}_{k}\right)}{\sqrt{\sum_{p \in P_{i, k}\left(r_{i, p}-\bar{r}_{i}\right)^{2}}} \sqrt{\sum_{p \in P_{i, k}}\left(r_{k, p}-\bar{r}_{k}\right)^{2}}},
$$

where $P_{i, k}$ indicates the totality of the products rated by the user $i$ and also the $k . \bar{r}_{i}$ user and $\bar{r}_{k}$ indicates the average ratings given by user $i$ and user $k$.

Constrained Pearson Correlation. The constrained Pearson correlation similarity, proposed by Shardanand and $\mathrm{Maes}^{48}$, is used when there are sufficient user data to provide recommendations, and there are multiple votes with the same rating, $r_{i, p}=\bar{r}_{i}$ :

$$
\operatorname{crs}_{i, k}=\frac{\sum_{p \in P_{i, k}}\left(r_{i, p}-r_{z}\right)\left(r_{k, p}-r_{z}\right)}{\sqrt{\sum_{p \in P_{i, k}}\left(r_{i, p}-r_{z}\right)^{2}} \sqrt{\sum_{p \in P_{i, k}}\left(r_{k, p}-r_{z}\right)^{2}}}
$$

where $r_{z}$ represents a neutral rating (neither likes nor dislikes) that in this specific case is represented by the constant 5 .

The second extraction management component is represented by the interesting items management component. This processes the results from the similarity measure management component. By calculating the probable interest level of the user to whom we want to provide recommendations, this component finds candidate products to which the user has not previously paid attention. For this purpose, it calculates the weighted average of similar users' ratings of these products as yet unknown to the user, using the similarity as weight. 


$$
\tilde{P}_{i, p}=\bar{r}_{i}+\frac{\sum_{k \in N} S_{i, k} \cdot\left(r_{k, p}-\bar{r}_{k}\right)}{\sum_{k \in N} S_{i, k}}
$$

where $\bar{r}_{i}$ is the user's $i$ e average ratings.

$$
S_{i, k}\left\{\begin{array}{c}
\operatorname{pip}_{i, k} \text { if Proximity Impact Popularity is applied } \\
r s_{i, k} \text { if Pearson Correlation is applied } \\
\operatorname{crs}_{i, k} \text { if Constrained Pearson Correlation is applied }
\end{array}\right.
$$

\section{INTERACTIONCOMMERCE IN PRACTICE}

Figure 3 represents the activity diagram in reference to the above-described architecture. Social activities are in gray background frames, while those connected to usual commercial transactions are in white background frames.

By observing the graphic, it is possible to monitor the flow of social commerce operations of Luigi Bianchi, a hypothetical customer, implemented according to the architectural specifications of Interaction Commerce. At the start of the path, Luigi can login in two different ways: through a "standard" registration, which includes e-mail verification and inputting user name and password, or through one of the social networks implemented by the platform. In the latter case, the user needs only to login through the social network, if not yet connected, and accept the authorizations necessary to automatically retrieve its social data. After login, Luigi can view comments and ratings entered by customers who have already made a purchase. It is important to note that, in this particular phase of the process, reading testimonials about their purchases can influence the path our customer follows. This functionality is managed by the architecture's transaction rating management component. Even if Luigi decides not to look at the comments and ratings, he still has the opportunity to view any product category managed by the catalogue management component or a list of products provided by the recommendation system management component. Practically, the customer can view specific products' categories of his choice, freely navigating within the social commerce, or relying upon the advice provided by the system about the products that interest him the most, by operating as if with a guide. Luigi's next step is to view all the characteristics of a specific product (using the product reviews management component), including the title, the price, and a description. During this phase, the customer can choose amongst various operations. The first operation allows Luigi to see the comments and ratings about a product posted by other users (managed by the reviews management component). By doing so, he can best evaluate his potential purchase. 
During this phase, Luigi can choose to post his comment about the product and return to viewing the comments left by other users, or he can choose to not post a comment or a rating and go back to viewing the product or category of products. Thanks to the social wish-list management component, the second operation is to add a product to a personal wish list. Once that is done, Luigi can go back to viewing other products to make other purchases or find information about his wish list and the items in it. As regards the wish list, Luigi can make different operations: delete products previously added to the list, view the details about a product on the wish list, or "convert" his wish list into an in-cart purchase. In that case, the customer purchases items he has been particularly interested in and were on his wish list. Once the "conversion" is done, Luigi can go back to viewing his wish list or his cart. The third operation Luigi can perform is to buy a product simply and immediately by placing the item in his cart (run by the cart management component) and remain on the same screen. Luigi can continue navigating through social commerce and make new purchases or view his cart (also run by the cart management component). Should Luigi decide to check his cart, he can change the quantity of each item he wishes to order or continue viewing a product and make other purchases. To confirm his order, Luigi can view the checkout information (run by the checkout management component). By this operation, the customer can decide not to finalize his purchase and go back to viewing the products, insert or change his purchase data such as his shipping and billing addresses or shipping and payment options, and confirm his order. If the transaction is carried out and the user deems it appropriate, he can add his rating and then comment about the transaction, so other users can read his comment before making a purchase. Luigi Bianchi's comment is important because it can be read by another customer and influence the choice to make or not make a purchase within the social commerce. 


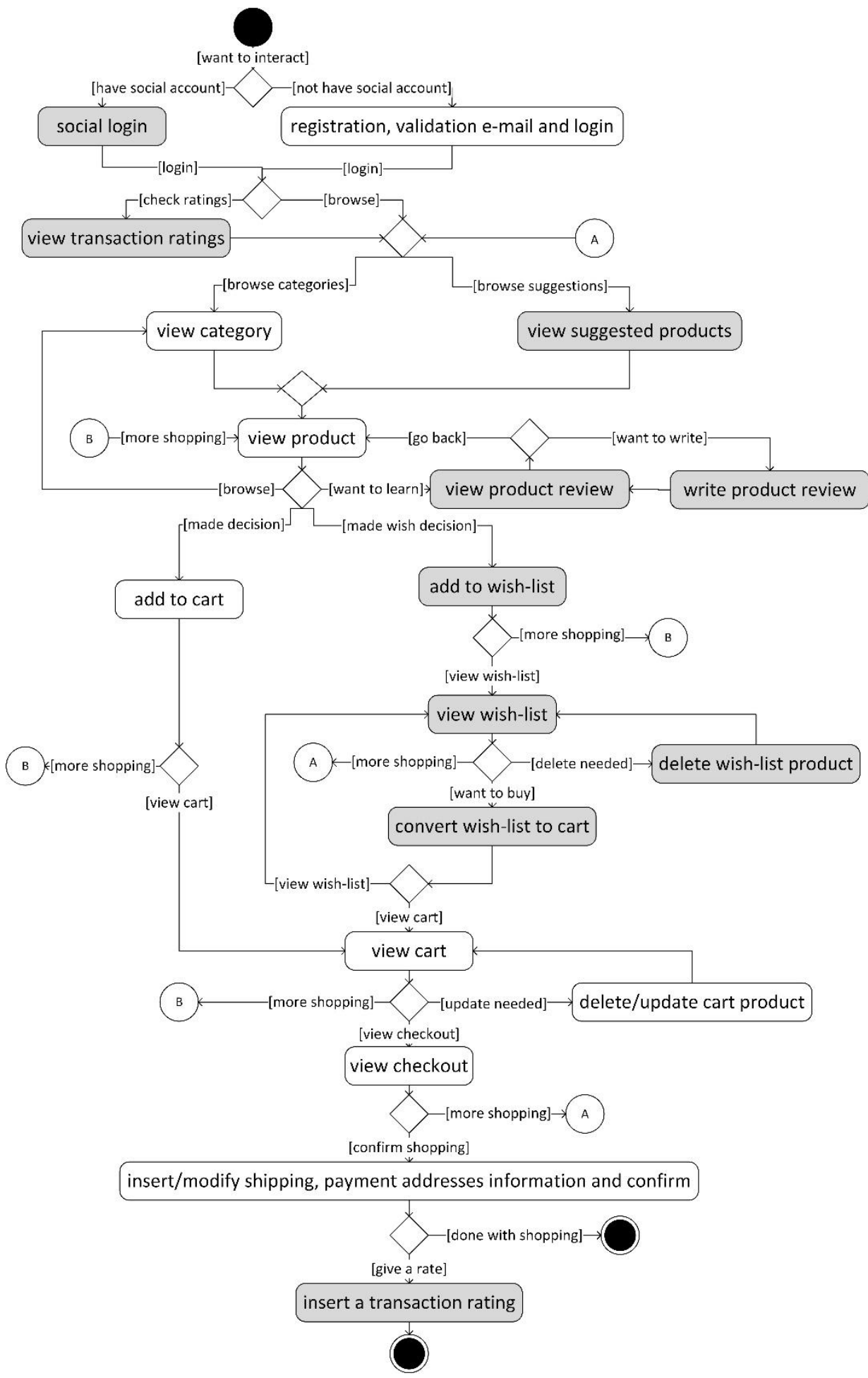

Figure 3. Interaction commerce activity diagram 


\section{EVALUATION}

This section is to validate the system discussed in this paper, paying particular attention to the architecture's most innovative component, which is the recommender system and its social approach. To validate the quality of the information offered by the system, we used a type of precision metric often used by information retrieval systems. This metric measures the system's ability to suggest content truly useful to the user. It can be calculated using the following formula:

$$
\text { Precision }=\frac{\text { correctly recommended content }}{\text { total } \text { recommended content }},
$$

where "correctly recommended content" is the group of recommendations of interest to the user and "total recommended content" is the total number of recommendations offered to the user.

Because it is subjective whether a recommendation is or is not of interest to a specific individual, it is not possible to automate the evaluation process. For this reason a group of 10 individuals, not expert in this field, were asked to evaluate the system by interacting with it. Specifically, they were asked to "like" the movies of interest to them from those listed on 15 Facebook pages and then login into e-commerce via the social network. Subsequently, within the Web portal (MovieLens dataset with 20 million ratings, which is publicly available at http://grouplens.org - was used), the subjects were offered 10 movies that were presumed to match their interests. Finally, they were asked to evaluate the recommendation and communicate which of the recommended movies were truly to their liking.

Figure 4 shows the number of movies imported from the social network onto the platform where the subjects had a positive reception because the user's social profile indicated interest. During the retrieval, some problems were found due to missing or wrong data coming from the social network, therefore related to the user. First, it was noticed how it was not possible to identify a user's interest for a specific movie if the movie was present on the social network but not in the shop's catalogue. Second, neither was it possible to determine if the user liked a movie, if the title of that movie found in the social network is different from the title of the movie in the shop (due, for example, to language differences). Third, because it is not possible to know from the social network when the movie was produced, it is possible to consider, which is more important for the user, other movies by the same title, produced at a different time, and having different contents. In spite of the problematic results just described, Figure 4 shows how it is possible to determine how the social preference acquisition component can be considered valid. The number of movies imported from 
the social network is substantial: considering the 10 tested individuals, the lowest number of imported movies is 12 , the average is 16.5 , and the highest is 22 .

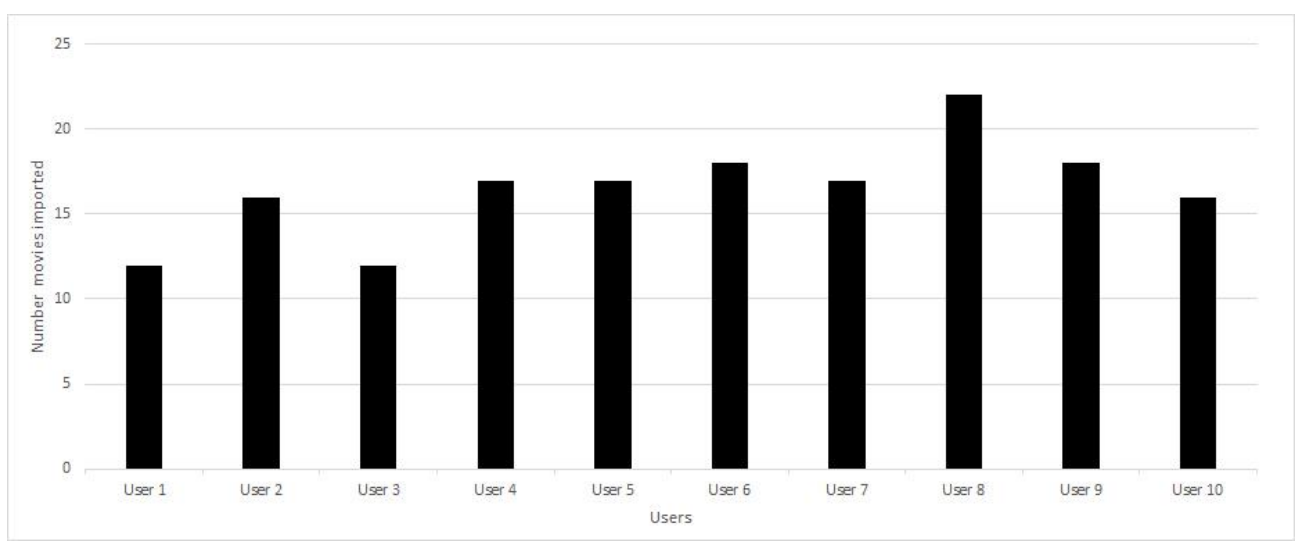

Figure 4. Number movies imported

Table 2 shows how many movies the tested subject considered interesting from amongst those recommended. By using these results it was possible to calculate the precision metric, as shown in Figure 5.

Table 2. Relevant movies through social network utilization

\begin{tabular}{ccc}
\hline User & No. suggested movies & No. pertinent movies \\
\hline 1 & 10 & 9 \\
2 & 10 & 5 \\
3 & 10 & 6 \\
4 & 10 & 5 \\
5 & 10 & 4 \\
6 & 10 & 2 \\
7 & 10 & 4 \\
8 & 10 & 6 \\
9 & 10 & 3 \\
10 & 10 & 6 \\
\hline
\end{tabular}

By studying the recommendations precision metric data, it is possible to make the prediction algorithm more effective, in terms of precision, for the user who is active on a social network and logs onto the social commerce platform network using his social profile. This is proved in Figure 5, showing that, of the 10 tested subjects, the average precision result is approximately 0.5 , with a 3.4 variance. Not taking into consideration 
users 1 and 6, who, respectively, show a high and low precision value, the users show a similar precision. Moreover, as shown in Table 3, should the users' social data not be available, the recommender system is forced to make random recommendations. This would increase the likelihood of offering the user wrong and irrelevant recommendations.

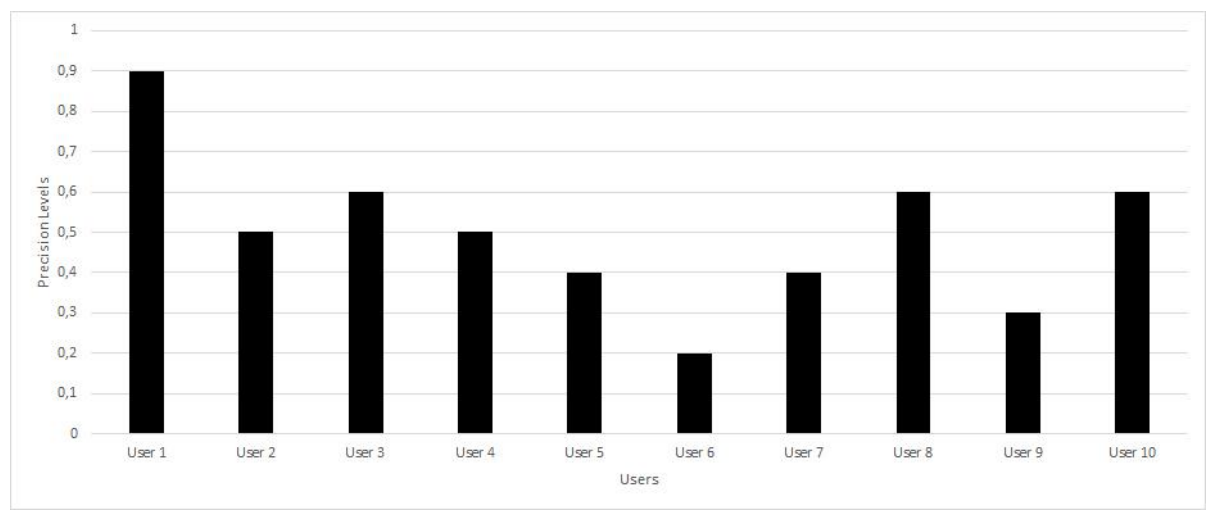

Figure 5. Precision

Table 3. Relevant movies without social network utilization

\begin{tabular}{ccc}
\hline User & $\begin{array}{c}\text { No. } \\
\text { suggested } \\
\text { movies }\end{array}$ & $\begin{array}{c}\text { No. pertinent } \\
\text { movies }\end{array}$ \\
1 & 10 & 3 \\
2 & 10 & 2 \\
3 & 10 & 5 \\
4 & 10 & 1 \\
5 & 10 & 2 \\
6 & 10 & 2 \\
7 & 10 & 4 \\
8 & 10 & 6 \\
9 & 10 & 2 \\
10 & 10 & 3 \\
\hline
\end{tabular}

\section{CONCLUSION}

This paper aims to describe the architecture of Interaction Commerce for social commerce both in its complexity and its singular components. There is a deep and detailed analysis of the modules' activities and the way each interfaces with the others. Particular attention is paid to the recommender system's management component. By focusing on that topic, 
we provide some components' definitions and describe the main approaches to the recommendation system. After a careful comparison of the various approaches, we explain our choice of using one algorithm instead of another. We also describe how the recommender system benefits by using social networks. By retrieving the user's information from a social network, after the user's first login, it is possible to limit, and sometimes even resolve, the cold-user start problem. This problem afflicts the majority, if not all, of recommendation algorithms and appears when a new user becomes part of social commerce, but the platform is not capable of recommending any products, not knowing the user's tastes. Last, by applying the architecture and testing a sample of users, it was possible to generate precision metrics of the recommendation algorithm when the user logs onto social networks and the platform is able to import data about its tastes. By using the generated metrics, it is possible to claim the correctness of the system-generated recommendations.

As to the presented work, we anticipate future tests in a variety of social commerce's domains and to optimize the similarity parameters for products available on the social network and those available in e-commerce catalogues. By doing so, it will be possible to correct any errors that might surface during the product matching phase.

In order to achieve a better targeted recommendation, we consider useful an in-depth study of the geographic origin of each platform user. Using social networks, it is possible to know the users' geographical location, and, adding such a parameter to the calculation of the users' similarities, we anticipate increased benefit from more precise recommendations. It remains to be seen whether, after more implementations and tests, this hypothesis will be confirmed or refuted.

\section{REFERENCES}

[1] C. Wang, and P. Zhang, The evolution of social commerce: The people, management, technology, and information dimensions. Communications of the Association for Information Systems, 31(5), 1-23, 2012.

[2] Y.R. Kang, and C. Park, Acceptance factors of social shopping. In M.-K. Choi (Ed.), Proceedings of 11th International Conference on Advanced Communication Technology (p2155-2159). Korea: IEEE Press, 2009.

[3] C.S.P. $\mathrm{Ng}$, Intention to purchase on social commerce websites across cultures: A cross-regional study. Information \& Management, 50(8), 609-620, 2013. http://dx.doi.org/10.1016/j.im.2013.08.002. 
[4] H. Ickler, S. Schülke, S. Wilfling, and U. Baumöl, New challenges in e-commerce: how social commerce influences the customer process. Paper presented at the 5th National Conference on Computing and Information Technology, Bangkok, Thailand, May 22-23, 2009.

[5] D. Kim, Under what conditions will social commerce business models survive? Electronic Commerce Research and Applications, 12(2), 69-77, 2013. http://dx.doi.org/10.1016/j.elerap.2012.12.002.

[6] R.G. Curty, and P. Zhang, Website features that gave rise to social commerce: a historical analysis. Electronic Commerce Research and Applications, 12(4), 260-279, 2013. http://dx.doi.org/10.1016/j.elerap.2013.04.001.

[7] Y.Y. Chen, F.W. Lai, K.N. Goh, and S.C. Daud. The effect of integrating social plugins into e-commerce website: A study on online consumer behaviour. Paper presented at the 7 th International Conference on Ubiquitous Information Management and Communication, Kota Kinabalu, Malaysia, January 17-19, 2013. http://dx.doi.org/10.1145/2448556.2448612.

[8] A.T. Stephen, and O. Toubia, Explaining the power-law degree distribution in a social commerce network. Social Networks, 31(4), 262-270, 2009. http://dx.doi.org/10.1016/j.socnet.2009.07.002.

[9] D. Kim, Process chain: A new paradigm of collaborative commerce and synchronized supply chain. Business Horizons, 49(5), 359-367, 2006. http://dx.doi.org/10.1016/j.bushor.2005.11.004.

[10] R. Burke, Integrating knowledge-based and collaborative-filtering recommender systems. Paper presented at the Workshop on AI and Electronic Commerce, Orlando, Florida, July 18-19, 1999.

[11] N.L.M. Shuib, N. Baiti, A.B. Normadhi, L.F.H.B.M. Alias, and N.S. Binti, Collaborative recommender system: A review. Paper presented at the International Conference on Advances in Computing, Electronics, and Electrical Technology, Kuala Lumpur, Malaysia, September 26-27, 2015.

[12] J. Lu, D. Wu, M. Mao, W. Wang, and G. Zhang, Recommender system application developments: A survey. Decision Support Systems, 74, 12-32, 2015. http://dx.doi.org/10.1016/j.dss.2015.03.008.

[13] Do. Goldberg, D. Nichols, B.M. Oki, and D. Terry. Using collaborative filtering to weave an information tapestry. Communications of the ACM, 35(12), 61-70, 1992. http://dx.doi.org/10.1145/138859.138867,

[14] G. Adomavicius, and A. Tuzhilin. Toward the next generation of recommender systems: A survey of the state-of-the-art and possible extensions. Knowledge and Data Engineering, 17(6), 734-749, 2005. http://dx.doi.org/10.1109/TKDE.2005.99,

[15] J. Bobadilla, F. Ortega, A. Hernando, and A. Gutiérrez, Recommender systems survey. Knowledge-Based Systems, 46, 109-132, 2013. 
http://dx.doi.org/10.1016/j.knosys.2013.03.012.

[16] S. Cleger-Tamayo, J.M. Fernández-Luna, and J.F. Huete, Top-N news recommendations in digital newspapers. Knowledge-Based Systems, 27, 180-189, 2012. http://dx.doi.org/10.1016/j.knosys.2011.11.017.

[17] D.H. Park, H.K. Kim, I.Y. Choi, and J.K. Kim, A literature review and classification of recommender systems research. Expert Systems with Applications, $\quad 39(11), \quad$ 10059-10072, 2012. http://dx.doi.org/10.1016/j.eswa.2012.02.038.

[18] S.M. Ali, and I. Ghani. A review on recommender techniques, systems and evaluation metrics. Science International Lahore, 24(4), 503-511, 2012.

[19] D. Cosley, S.K. Lam, I. Albert, J.A. Konstan, and J. Riedl. Is seeing believing? How recommender system interfaces affect users' opinions. Paper presented at the SIGCHI Conference on Human Factors in Computing Systems, Ft. Lauderdale, Florida, USA, April 5-10, 2003. http://dx.doi.org/10.1145/642611.642713.

[20] M.J. Pazzani, and D. Billsus, Content-based recommendation systems. In P. Brusilovsky, A, Kobsa, and W. Nejdl (Eds.), The Adaptive Web (p325-341). Berlin, Heidelberg, New York: Springer, 2007. http://dx.doi.org/10.1007/978-3-540-72079-9_10.

[21] A. Kobsa, User modeling and user-adapted interaction. Paper presented at the Conference Companion on Human Factors in Computing Systems, Boston, Massachusetts, USA, April 24-28, 1994. https://doi.org/10.1145/259963.260532.

[22] C. Basu, H. Hirsh, and W. Cohen, Recommendation as classification: Using social and content-based information in recommendation. Paper presented at the Fifteenth National/Tenth Conference on Artificial Intelligence/Innovative Applications of Artificial Intelligence, Madison, Wisconsin, USA, July 26-30, 1998.

[23] D. Mladenic. Text-learning and related intelligent agents: A survey. IEEE Intelligent Systems, 14(4), 44-54, 1999. http://dx.doi.org/10.1109/5254.784084.

[24] P. Lops, M. De Gemmis, and G. Semeraro. Content-based recommender systems: State of the art and trends. In F. Ricci, L. Rokach, B. Shapira, and Paul B. Kantor (Eds.), Recommender Systems Handbook (p73-105). Berlin, Heidelberg, New York: Springer, 2011. http://dx.doi.org/10.1007/978-0-387-85820-3_3.

[25] M. Deshpande, and G. Karypis, Item-based top-n recommendation algorithms. ACM Transactions on Information Systems (TOIS), 22(1), 143-177, 2004. http://dx.doi.org/10.1145/963770.963776.

[26] J.L. Herlocker, J.A. Konstan, L.G. Terveen, and J.T. Riedl, Evaluating collaborative filtering recommender systems. ACM Transactions on Information Systems (TOIS), 22(1), 5-53, 2004. 
http://dx.doi.org/10.1145/963770.963772.

[27] L. Candillier, F. Meyer, and M. Boullé. Comparing state-of-the-art collaborative filtering systems. In P. Perner (Ed.), Machine Learning and Data Mining in Pattern Recognition (p548-562). Berlin, Heidelberg, New York: Springer, 2007. http://dx.doi.org/10.1007/978-3-540-73499-4_41.

[28] J.L. Herlocker, J.A. Konstan, A. Borchers, and J. Riedl, An algorithmic framework for performing collaborative filtering. Paper presented at the 22nd Annual International ACM SIGIR Conference on Research and Development in Information Retrieval, Berkeley, California, USA, August 15-19, 1999. https://doi.org/10.1145/312624.312682.

[29] J.L. Herlocker, J.A. Konstan, and J. Riedl, Explaining collaborative filtering recommendations. Paper presented at the 2000 ACM Conference on Computer Supported Cooperative Work, Philadelphia, PA, USA, December 02-06, 2000. http://dx.doi.org/10.1145/358916.358995.

[30] X. Su, and T. M. Khoshgoftaar. A survey of collaborative filtering techniques. Advances In Artificial Intelligence, Section 3, 1-20, 2009. http://dx.doi.org/10.1155/2009/421425.

[31] J. Bobadilla, A. Hernando, F. Ortega, and A. Gutiérrez, Collaborative filtering based on significances. Information Sciences, 185(1), 1-17, 2012. http://dx.doi.org/10.1016/j.ins.2011.09.014.

[32] J. Bobadilla, A. Hernando, F. Ortega, and J. Bernal, A framework for collaborative filtering recommender systems. Expert Systems with Applications, $\quad 38(12), \quad$ 14609-14623, 2011. http://dx.doi.org/10.1016/j.eswa.2011.05.021.

[33] M. Ghazanfar, and A. Prugel-Bennett, Fulfilling the needs of gray-sheep users in recommender systems, a clustering solution. Paper presented at the International Conference on Information Systems and Computational Intelligence, Harbin, China, January 18-20, 2011.

[34] S.T. Park, and W. Chu, Pairwise preference regression for cold-start recommendation. Paper presented at the Third ACM Conference on Recommender Systems, New York, USA, October 23-25, 2009. http://dx.doi.org/10.1145/1639714.1639720.

[35] G. Linden, B. Smith, and J. York. Amazon.com recommendations: Item-to-item collaborative filtering. Internet Computing, 7(1), 76-80, 2003. http://dx.doi.org/10.1109/MIC.2003.1167344.

[36] T. Hofmann. Latent semantic models for collaborative filtering. ACM Transactions on Information Systems (TOIS), 22(1), 89-115, 2004. http://dx.doi.org/10.1145/963770.963774.

[37] R. Van Meteren, and M. Van Someren, Using content-based filtering for recommendation. Paper presented at the Machine Learning in the New Information Age: MLnet/ECML2000 Workshop, Barcelona, 
Catalonia, Spain, May 30, 2000.

[38] K. Sparck Jones, A statistical interpretation of term specificity and its application in retrieval. Journal of documentation, 28(1), 11-21, 1972. http://dx.doi.org/10.1108/eb026526.

[39] J. Salter, and N. Antonopoulos, CinemaScreen recommender agent: combining collaborative and content-based filtering. Intelligent Systems, 21(1), 35-41, 2006. http://dx.doi.org/10.1109/MIS.2006.4.

[40] L. Quijano-Sánchez, D. Bridge, B. Díaz-Agudo, and J.A. Recio-García, A case-based solution to the cold-start problem in group recommenders. In B.D. Agudo and I. Watson (Eds.), A Case-Based Reasoning Research and Development (p342-356). Berlin, Heidelberg, New York: Springer, 2012. http://dx.doi.org/10.1007/978-3-642-32986-9_26.

[41] K. Yu, A. Schwaighofer, V. Tresp, X. Xu, and H.P. Kriegel, Probabilistic memory-based collaborative filtering. Knowledge and Data Engineering, 16(1), 56-69, 2004. http://dx.doi.org/10.1109/TKDE.2004.1264822.

[42] J.B. Schafer, D. Frankowski, J. Herlocker, and S. Sen, Collaborative filtering recommender systems. In P. Brusilovsky, A. Kobsa, and W. Nejdl (Eds.), The Adaptive Web (p 291-324). Berlin, Heidelberg, New York: Springer, 2007. http://dx.doi.org/10.1007/978-3-540-72079-9_9.

[43] J. Bobadilla, F. Ortega, A. Hernando, and J. Bernal, A collaborative filtering approach to mitigate the new user cold start problem. Knowledge-Based Systems, 26, 225-238, 2012. http://dx.doi.org/10.1016/j.knosys.2011.07.021.

[44] A.I. Schein, A. Popescul, L.H. Ungar, and D.M. Pennock, Methods and metrics for cold-start recommendations. Paper presented at the 25th Annual International ACM SIGIR Conference on Research and Development in Information Retrieval, Tampere, Finland, August 11-15, 2002. http://dx.doi.org/10.1145/564376.564421.

[45] A.M. Rashid, G. Karypis, and J. Riedl, Learning preferences of new users in recommender systems: An information theoretic approach. ACM SIGKDD Explorations Newsletter, 10(2), 90-100, 2008. http://dx.doi.org/10.1145/1540276.1540302.

[46] P. du Boucher-Ryan, and D. Bridge, Collaborative recommending using formal concept analysis. Knowledge-Based Systems, 19(5), 309-315, 2006. http://dx.doi.org/10.1016/j.knosys.2005.11.017.

[47] H.J. Ahn, A new similarity measure for collaborative filtering to alleviate the new user cold-starting problem. Information Sciences, 178(1), 37-51, 2008. http://dx.doi.org/10.1016/j.ins.2007.07.024.

[48] U. Shardanand, and P. Maes, Social information filtering: algorithms for automating "word of mouth". Paper presented at the SIGCHI Conference on Human Factors in Computing Systems, Denver, 
Colorado, USA, $\quad$ May 1995. https://doi.org/10.1145/223904.223931. 